\title{
Efficacy of alemtuzumab over 6 years in relapsing-remitting multiple sclerosis patients who relapsed between courses 1 and 2: Post hoc analysis of the CARE-MS studies
}

Multiple Sclerosis Journal

$1-10$

DOI: 10.1177

1352458519881759

(C) The Author(s), 2019 (c) (i) (5)

Article reuse guidelines: sagepub.com/journalspermissions

\author{
Bart Van Wijmeersch, Barry A Singer, Aaron Boster, Simon Broadley (iD, \\ Óscar Fernández, Mark S Freedman, Guillermo Izquierdo, Jan Lycke, Carlo Pozzilli, \\ Basil Sharrack, Brian Steingo, Heinz Wiendl, Sibyl Wray, Tjalf Ziemssen (D), \\ Luke Chung, David H Margolin, Karthinathan Thangavelu and Patrick Vermersch; \\ on behalf of the CARE-MS I, CARE-MS II, and CAMMS03409 Investigators
}

\section{Abstract}

Background: Alemtuzumab is administered as two annual courses for relapsing-remitting multiple sclerosis (MS). Patients may relapse before completing the two-course regimen.

Objective: The objective was to evaluate 6-year outcomes in patients who relapsed between alemtuzumab Courses 1 and 2 (early relapsers).

Methods: Post hoc analysis of patients from the Comparison of Alemtuzumab and Rebif ${ }^{\circledR}$ Efficacy in Multiple Sclerosis (CARE-MS) studies who enrolled in the extension.

Results: Early relapsers (CARE-MS I: 15\%; CARE-MS II: 24\%) had more relapses in 1-2 years prealemtuzumab and higher mean baseline Expanded Disability Status Scale score than patients without relapse. Their annualized relapse rate declined from Year 1 (CARE-MS I: 1.3; CARE-MS II: 1.2) to Year 2 following Course $2(0.3 ; 0.5)$ and remained low thereafter. Over 6 years, $60 \%$ remained free of 6-month confirmed disability worsening; 24\% (CARE-MS I) and 34\% (CARE-MS II) achieved 6-month confirmed disability improvement. During Year 6, 69\% (CARE-MS I) and 68\% (CARE-MS II) were free of magnetic resonance imaging (MRI) disease activity. Median percent yearly brain volume loss (Year 1: $-0.67 \%$ (CARE-MS I); $-0.47 \%$ (CARE-MS II)) declined after Course 2 (Year $6:-0.24 \% ;-0.13 \%$ ).

Conclusion: Early relapsers' outcomes improved after completing the second alemtuzumab course. These findings support administering the approved two-course regimen to maximize clinical benefit.

ClinicalTrials.gov registration numbers: CARE-MS I, II, extension: NCT00530348, NCT00548405, NCT00930553.

Keywords: Alemtuzumab, disease-modifying therapy, relapsing-remitting multiple sclerosis (MS), efficacy, magnetic resonance imaging (MRI), disability

Date received: 15 March 2019; revised: 22 August 2019; accepted: 23 August 2019

\section{Introduction}

Two alemtuzumab courses significantly improved efficacy outcomes versus subcutaneous interferon beta-1a (SC IFNB-1a) in patients with active relapsing-remitting multiple sclerosis (RRMS) in two phase 3 trials (Comparison of Alemtuzumab and Rebif ${ }^{B}$ Efficacy in Multiple Sclerosis (CARE-MS) I and II). ${ }^{1,2}$ In the CARE-MS extension study, efficacy was maintained through Year 6, including improvements in preexisting disability and significant slowing of brain volume loss (BVL), ${ }^{3-6}$ with 64\% (CARE-MS I) and $55 \%$ (CARE-MS II) of patients not receiving additional alemtuzumab after the initial two courses. ${ }^{6}$ The effects of alemtuzumab over 6 years in the absence of continuous treatment may be due to its selective depletion and distinct pattern of repopulation of circulating CD52-expressing T and B lymphocytes. ${ }^{7-9}$ Adverse events (AEs) associated with alemtuzumab treatment in clinical trials and postmarketing experience include infusion-associated reactions (IARs),
Correspondence to

B Van Wijmeersch

Faculty of Medicine and

Life Sciences, Hasselt

University, Campus Hasselt,

Martelarenlaan 42, 3500

Hasselt, Belgium.

bart.vanwijmeersch@

uhasselt.be

Bart Van Wijmeersch Rehabilitation and MS

Center Overpelt; BIOMED,

Hasselt University, Hasselt,

Belgium

Barry A Singer

The MS Center for

Innovations in Care, Missour

Baptist Medical Center, St

Louis, MO, USA

Aaron Boster

OhioHealth Neurological

Physicians, Columbus, $\mathrm{OH}$,

USA

Simon Broadley

School of Medicine, Griffith

University, Southport, QLD,

Australia

Óscar Fernández Fundación IMABIS, Hospital

Universitario Carlos Haya,

Málaga, Spain

Mark S Freedman

University of Ottawa and The Ottawa Hospital Research

Institute, Ottawa, ON,

Canada

Guillermo Izquierdo

Virgen Macarena University Hospital, Seville, Spain

Jan Lycke

Institution of Neuroscience and Physiology, Sahlgrenska Academy, University of Gothenburg, Gothenburg, Sweden

Carlo Pozzilli

Sapienza University of Rome, Rome, Italy

Basil Sharrack

NIHR Sheffield Biomedical 
Research Centre, Sheffield

Teaching Hospitals,

University of Sheffield,

Sheffield, UK

Brian Steingo

Fort Lauderdale Multiple

Sclerosis Center, Fort

Lauderdale, FL, USA

Heinz Wiend

University of Münster,

Münster, Germany

Sibyl Wray

Hope Neurology PLLC,

Knoxville, TN, USA

Tjalf Ziemssen

Center of Clinical

Neuroscience, Carl Gustav

Carus University Hospital,

Dresden, Germany

Luke Chung

Sanofi, Cambridge, MA,

USA

David H Margolin

Sanofi, Cambridge, MA,

USA; Cerevance, Boston,

MA, USA

Karthinathan Thangavelu

Sanofi, Cambridge, MA,

USA; EMD Serono, Billerica, MA, USA

Patrick Vermersch

University of Lille, INSERM

U995, CHU Lille, FHU

Imminent, Lille, France increased frequency of infection and the potential for opportunistic infections, secondary autoimmunity (thyroid disorders, immune thrombocytopenia, nephropathies, autoimmune cytopenias, autoimmune hepatitis, and other less common autoimmune events), acute acalculous cholecystitis, and cardiovascular and pulmonary events possibly related to infusion. ${ }^{1,2,4-6,10-13}$

Alemtuzumab is administered as two courses at $12 \mathrm{mg} /$ day intravenously on five consecutive days at baseline and three consecutive days 12 months later. ${ }^{11,14}$ Product labeling allows for additional courses as needed in certain countries, for example, in the United States (no limit) ${ }^{14}$ and in Australia and Europe (up to 2). ${ }^{11,15}$ As with other disease-modifying therapies (DMTs), ${ }^{16-18}$ some alemtuzumab-treated patients may relapse within the first year of therapy. An important question is whether patients relapsing between the first and second alemtuzumab courses can expect to achieve control of disease activity after completion of the second course. In this post hoc analysis, 6-year clinical and magnetic resonance imaging (MRI) outcomes were assessed in CARE-MS alemtuzumab-treated patients who relapsed between Courses 1 and 2 (referred to here as "early relapsers") and in those patients without early relapse ("early non-relapsers").

\section{Materials and methods}

\section{Patients and procedures}

This post hoc analysis included patients who received alemtuzumab in the core CARE-MS I and II trials (NCT00530348; NCT00548405), subsequently entered the extension study (CAMMS03409; NCT00930553), and had up to 6 years of follow-up after initiating alemtuzumab (2 years in the core study and 4 years in the extension). ${ }^{1,2,4,5}$ Patient subgroups were defined according to relapse in Months 0-12 (between Courses 1 and 2).

The study designs for the CARE-MS core and extension studies were published previously. ${ }^{1,2,4,5}$ Briefly, CARE-MS I and II were randomized, rater-blinded, active-controlled, head-to-head trials of alemtuzumab $(12 \mathrm{mg} /$ day intravenously on five consecutive days at baseline and three consecutive days 12 months later) compared with SC IFNB-1a ( $44 \mu \mathrm{g}$ three times per week) in patients with active RRMS ( $\geqslant 2$ relapses in the previous 2 years and $\geqslant 1$ relapse in the prior year) who were treatment naive (CARE-MS I) ${ }^{1}$ or had inadequate response to prior therapy with IFNB or glatiramer acetate (CARE-MS II). ${ }^{2}$ Patients completing the core studies could enroll in the extension and receive additional alemtuzumab (12 $\mathrm{mg} /$ day intravenously on three consecutive days), as needed for disease activity, $\geqslant 12$ months after the previous infusion. ${ }^{4,5}$ Criteria for additional courses were $\geqslant 1$ protocol-defined relapse, or $\geqslant 2$ new/enlarging $\mathrm{T} 2$ hyperintense and/or new gadolinium (Gd)-enhancing T1 brain or spinal cord lesions on MRI; investigators had discretion whether to administer additional courses to qualifying patients. Use of other DMTs was permitted during the extension at investigator's discretion (no criteria). Patients who received SC IFNB-1a in the core CARE-MS studies and who switched to alemtuzumab were not included in this analysis.

All procedures were approved by local institutional ethics review boards of participating sites. Patients provided written informed consent. ${ }^{4,5}$

\section{Outcome measures}

Patient characteristics were assessed at baseline. Details regarding relapse assessment, Expanded Disability Status Scale (EDSS) scores, and MRI scans were described previously; ${ }^{4-6}$ details on the MRI methodology are shown in the Supplementary Material. Post hoc subgroup analyses over 6 years included the following: annualized relapse rate (ARR); 6-month confirmed disability worsening (CDW; $\geqslant 1.0$-point EDSS score increase from core study baseline $(\geqslant 1.5$ if baseline EDSS score=0)); 6-month confirmed disability improvement (CDI; $\geqslant 1.0$-point decrease from the core study baseline EDSS score, in patients with baseline EDSS scores $\geqslant 2.0$ ); absence of MRI disease activity (new Gd-enhancing and new/enlarging T2 hyperintense lesions); and median percentage BVL, derived by relative change in brain parenchymal fraction (BPF; calculated from proton density/ T2-weighted dual-echo images using brain segmentation software developed at the Cleveland Clinic (Cleveland, $\mathrm{OH})$ ). Analyses also included proportions of patients with MRI lesions (Gd-enhancing, new/ enlarging T2 hyperintense, and new non-enhancing T1 hypointense lesions) and no evidence of disease activity (NEDA, absence of both clinical disease activity (relapses and 6-month CDW) and MRI disease activity).

\section{Statistical analysis}

Analyses were based on available data (without imputation) on all patients administered with alemtuzumab $12 \mathrm{mg}$ up to 6 years of follow-up from the first dose in CARE-MS I or II. Comparison of baseline characteristics between early relapsers and early non-relapsers 


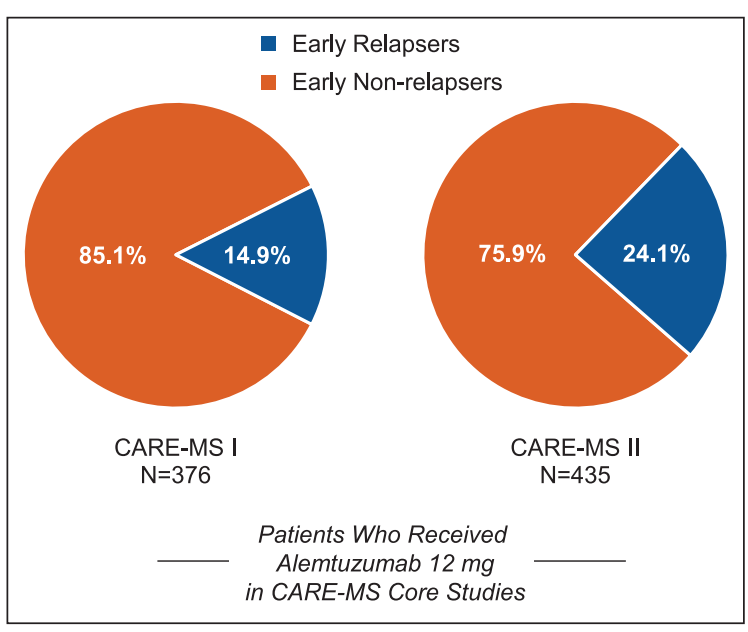

Figure 1. Proportions of early relapsers and early nonrelapsers. Proportions of patients with and without relapse between alemtuzumab Courses 1 and 2 in the core CAREMS I (left) and CARE-MS II (right) studies.

CARE-MS: Comparison of Alemtuzumab and Rebif ${ }^{\mathbb{E}}$ Efficacy in Multiple Sclerosis.

was done using the chi-square test for categorical variables and the Wilcoxon rank-sum test for continuous variables. The $p$ values obtained were nominal and not adjusted for multiple comparisons.

ARR was estimated using negative binomial regression with robust variance estimation. Proportions of patients with 6-month CDW or 6-month CDI were estimated with the Kaplan-Meier method. Yearly percentage BPF changes and proportions of patients free of Gd-enhancing, new T1 hypointense, or new/enlarging T2 hyperintense lesions were reported. NEDA and freedom from MRI disease activity were evaluated annually.

\section{Results}

\section{5\%-24\% of patients relapsed between Courses 1} and 2 of alemtuzumab

Of 376 patients who received alemtuzumab $12 \mathrm{mg}$ in CARE-MS I, 56 (15\%) relapsed within 12 months after treatment initiation; a higher percentage of early relapsers was observed among CARE-MS II patients (105/435 (24\%) Figure 1 and eFigure 1 (in Supplementary Material)). In comparison, 32\% (CARE-MS I) and 43\% (CARE-MS II) of SC IFNB-1 a-treated patients relapsed within the first year after IFNB-1a initiation. Early relapses in alemtuzumab-treated patients occurred in a mean of 6.3 (standard deviation (SD), 3.5) months after Course 1 in CARE-MS I and 5.5 (3.5) months in CARE-MS II, with $59 \%$ and $49 \%$ of early relapsers experiencing relapse in Months 7-12 only. Retention rates from core study baseline through the end of Year 6 were $80 \%$ (CARE-MS I) and 72\% (CARE-MS II) for early relapsers and $88 \%$ (CARE-MS I) and $81 \%$ (CARE-MS II) for early non-relapsers.

Through the 4-year extension study, 46\% (CARE-MS I) and 60\% (CARE-MS II) of early relapsers received a third or subsequent courses of alemtuzumab. The mean time between Courses 2 and 3 was 1.9 years (CARE-MS I) and 2.0years (CARE-MS II). The mean time between Courses 3 and 4 was 1.5 years (CARE-MS I) and 1.4 years (CARE-MS II). In the early non-relapse group, fewer patients received additional courses (CARE-MS I: 34\%, CARE-MS II: $41 \% ; p<0.05$ vs. early relapsers), with mean 2.3 years (CARE-MS I) and 2.4 years (CARE-MS II) between Courses 2 and 3 and 1.9years (both studies) between Courses 3 and 4 . Proportions of early relapsers who enrolled into the extension study and received a total of three courses (CARE-MS I: 21\%; CARE-MS II: $33 \%$ ) were greater than those receiving a total of four courses (CARE-MS I: 17\%; CARE-MS II: $20 \%$ ) or greater than four courses (CARE-MS I: 8\%; CARE-MS II: 7\%); 5\% of CARE-MS II early relapsers were treated every year (i.e. received six courses).

\section{Early relapsers had increased baseline disease activity}

Before alemtuzumab treatment, early relapsers from each CARE-MS study had a significantly higher mean EDSS score than early non-relapsers and significantly more relapses in the prior 1-2 years (Table 1). The CARE-MS I early relapse group also had a significantly higher percentage of female patients.

Efficacy outcomes in early relapsers after Course 2 Among early relapsers, 96\% (CARE-MS I) and 95\% (CARE-MS II) received Course 2. After the second course, $71 \%$ (CARE-MS I; 95\% confidence interval (CI): 59.6\%, 83.3\%) and 59\% (CARE-MS II; 49.6\%, $68.4 \%$ ) of patients were free of relapse in Year 2, and ARR declined from Year 1 to Year 2 by $74 \%$ in CARE-MS I and by 56\% in CARE-MS II, remaining low in Years 3-6 (Figure 2(a)). Over 6years, $60 \%$ of patients in each study ( $95 \%$ CI: $45.0 \%, 71.4 \%$; $48.4 \%$, $69.3 \%$ ) were free of 6-month CDW and 24\% (CARE-MS I; $13.9 \%, 40.6 \%$ ) and $34 \%$ (CARE-MS II; $24.4 \%$, 47.1\%) achieved 6-month CDI (Figure 2(b) and (c)).

Despite relapse in the first year, $82 \%$ (CARE-MS I) and $79 \%$ (CARE-MS II) of patients were free of Gd-enhancing lesions, $54 \%$ and $56 \%$ were free of 
Table 1. Core study baseline characteristics of early relapsers and early non-relapsers.

\begin{tabular}{|c|c|c|c|c|}
\hline \multirow[t]{2}{*}{ Parameters } & \multicolumn{2}{|l|}{ CARE-MS I } & \multicolumn{2}{|l|}{ CARE-MS II } \\
\hline & $\begin{array}{l}\text { Early Relapsers } \\
(n=56)\end{array}$ & $\begin{array}{l}\text { Early Non-relapsers } \\
(n=320)\end{array}$ & $\begin{array}{l}\text { Early Relapsers } \\
(n=105)\end{array}$ & $\begin{array}{l}\text { Early Non-relapsers } \\
(n=330)\end{array}$ \\
\hline Age, years, mean (SD) & $33.6(7.4)$ & $32.9(8.2)$ & $34.6(8.8)$ & $34.8(8.2)$ \\
\hline Female, $n(\%)$ & $43(77)$ & $200(63)^{\mathrm{a}}$ & $77(73)$ & $210(64)$ \\
\hline White, $n(\%)$ & $49(88)$ & $303(95)$ & $96(91)$ & $296(90)$ \\
\hline EDSS score, mean (SD) & $2.3(0.7)$ & $2.0(0.8)^{\mathrm{a}}$ & $3.0(1.4)$ & $2.6(1.2)^{\mathrm{a}}$ \\
\hline $\begin{array}{l}\text { Years since the initial } \\
\text { clinical attack, mean (SD) }\end{array}$ & $1.7(1.2)$ & $2.2(1.4)$ & $4.3(2.7)$ & $4.5(2.7)$ \\
\hline $\begin{array}{l}\text { Years since the last } \\
\text { relapse, mean (SD) }\end{array}$ & $0.3(0.2)$ & $0.4(0.2)$ & $0.4(0.2)$ & $0.4(0.2)$ \\
\hline $\begin{array}{l}\text { No. of relapses in prior } \\
1 \text { year, mean }(\mathrm{SD})\end{array}$ & $2.18(0.90)$ & $1.69(0.78)^{\mathrm{a}}$ & $1.85(0.90)$ & $1.60(0.85)^{\mathrm{a}}$ \\
\hline $\begin{array}{l}\text { No. of relapses in prior } \\
2 \text { years, mean (SD) }\end{array}$ & $2.91(0.88)$ & $2.45(0.83)^{\mathrm{a}}$ & $3.14(1.37)$ & $2.65(1.10)^{\mathrm{a}}$ \\
\hline $\begin{array}{l}\text { Gd-enhancing lesion } \\
\text { count, mean (SD) }\end{array}$ & $2.4(4.5)$ & $2.3(5.2)$ & $1.6(3.1)$ & $2.5(6.7)$ \\
\hline $\begin{array}{l}\text { Patients with Gd- } \\
\text { enhancing lesions, } n(\%)\end{array}$ & $25(44.6)$ & $146(46.3)$ & $41(39.0)$ & $140(43.2)$ \\
\hline $\mathrm{BPF}$, mean $(\mathrm{SD})$ & $0.82(0.02)$ & $0.82(0.02)$ & $0.82(0.02)$ & $0.81(0.02)$ \\
\hline
\end{tabular}

new/enlarging T2 hyperintense lesions, 71\% and 80\% were free of new non-enhancing $\mathrm{T} 1$ hypointense lesions, and $54 \%$ and $55 \%$ were free of MRI disease activity during that year (Figure 3(a) and eTable 1 (in Supplementary Material)). Annually through Years $2-6,64 \%-77 \%$ remained free of MRI disease activity. In each year after Course 2, 48\%-59\% (CARE-MS I) and $38 \%-58 \%$ (CARE-MS II) of early relapsers achieved annual NEDA (eTable 1). The percentages of early relapsing patients achieving cumulative NEDA through the 4-year extension study (Years 3-6) were 27\% (CARE-MS I) and 16\% (CARE-MS II).

Median percentage yearly BPF change in early relapsers declined markedly after Course 2 (CARE-MS I: Year $1,-0.67 \%$ (95\% CI: $-1.10 \%,-0.39 \%)$ vs. Year $2,-0.17 \%(-0.39 \%, 0 \%)$; CARE-MS II: Year 1, $-0.47 \% \quad(-0.61 \%,-0.24 \%)$ vs. Year 2, $-0.10 \%$ $(-0.25 \%, 0.05 \%))$ and remained low over the extension Years 3-6 (Figure 3(b)).

\section{Efficacy outcomes in early non-relapsers through 6 years}

Among early non-relapsers, 92\% (CARE-MS I) and $88 \%$ (CARE-MS II) were relapse-free during Year 6 and ARR remained low after Course 2 through 6 years
(eTable 2 in Supplementary Material). Over 6 years, 80\% (CARE-MS I; 95\% CI: 75.1\%, 84.3\%) and 75\% (CARE-MS II; 69.5\%, 79.4\%) of patients were free of 6-month CDW, and 36\% (CARE-MS I; 29.4\%, $43.8 \%$ ) and $45 \%$ (CARE-MS II; 39.1\%, 52.1\%) achieved 6-month CDI (eTable 2).

In each year through Year 6, 61\%-78\% (CARE-MS I) and $63 \%-78 \%$ (CARE-MS II) of patients were free of MRI disease activity, and $59 \%-70 \%$ and $56 \%-64 \%$ achieved annual NEDA (eTable 2). Through the extension Years 3-6, 34\% (CARE-MS I) and 24\% (CARE-MS II) of early non-relapsers achieved cumulative NEDA. Median percentage yearly BPF change decreased over 6 years in early non-relapsers who had enrolled in the extension study from CARE-MS I (Year 1: $-0.57 \%(95 \% \mathrm{CI}:-0.75 \%,-0.51 \%)$ to Year 6: $-0.16 \%(-0.25 \%,-0.09 \%))$ or CARE-MS II (Year 1: $-0.49 \%(-0.58 \%,-0.39 \%)$ to Year $6:-0.10 \%$ $(-0.19 \%, 0 \%)$; eTable 2$)$.

Early relapsers showed moderately elevated relapse risk and disability worsening

In Year 6, ARR was comparable between early relapsers and early non-relapsers in CARE-MS II but was slightly elevated in CARE-MS I early relapsers 
(a)

CARE-MS I Early Relapsers

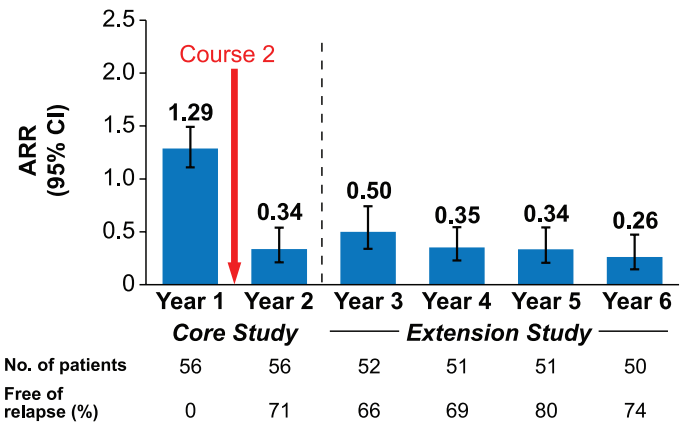

(b)

\section{CARE-MS I Early Relapsers}

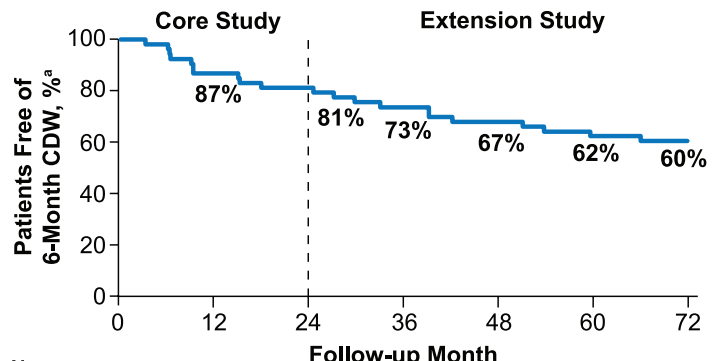

No.

$\begin{array}{llllllll}\text { at risk } & 52 & 45 & 42 & 38 & 35 & 32 & 27\end{array}$

(c)

\section{CARE-MS I Early Relapsers}

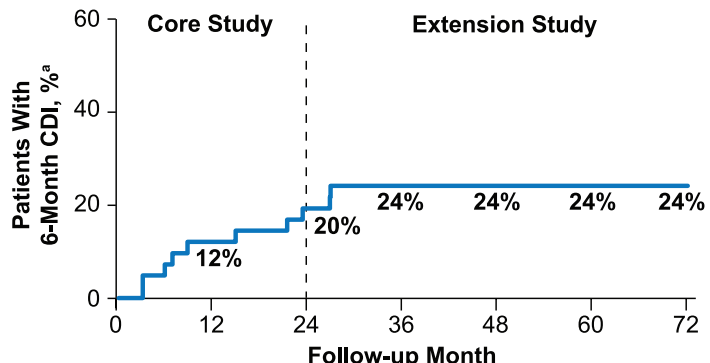

No.

$\begin{array}{llllllll}\text { at risk }^{\mathrm{b}} & 41 & 36 & 33 & 31 & 31 & 30 & 24\end{array}$
CARE-MS II Early Relapsers

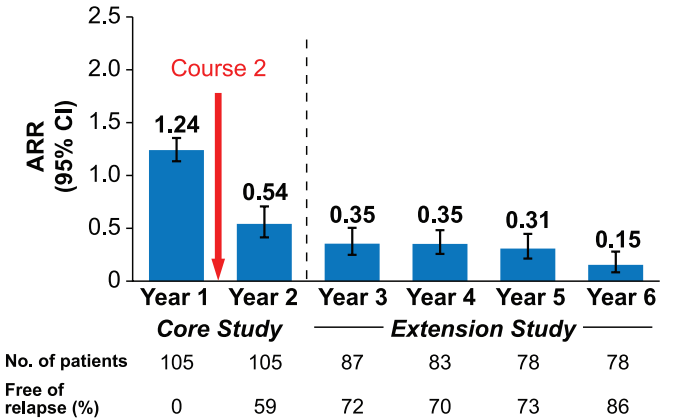

\section{CARE-MS II Early Relapsers}

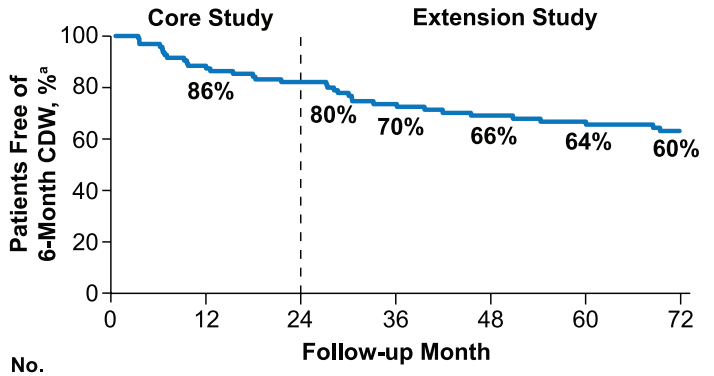

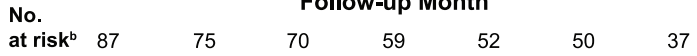

CARE-MS II Early Relapsers

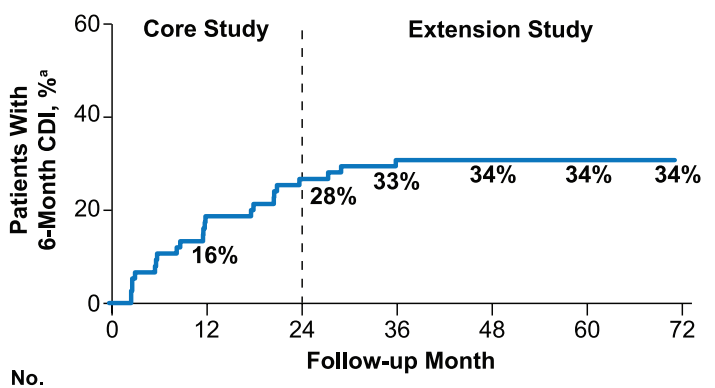

Figure 2. Clinical efficacy outcomes in early relapsers through 6 years. (a) ARR and proportions free of relapse, (b) proportions free of 6-month CDW, and (c) proportions achieving 6-month CDI over 6 years in patients with relapse between alemtuzumab Courses 1 and 2 in the core CARE-MS I and CARE-MS II studies.

ARR: annualized relapse rate; CARE-MS: Comparison of Alemtuzumab and Rebif ${ }^{\text {E }}$ Efficacy in Multiple Sclerosis; CDI: confirmed disability improvement; CDW: confirmed disability worsening; CIs: confidence intervals; EDSS: Expanded Disability Status Scale. Error bars denote $95 \%$ CIs.

aKaplan-Meier estimates.

${ }^{b}$ Number at risk is the number of patients who remained on study and had yet to experience 6-month CDW or 6-month CDI. CDI is defined as $\geqslant 1$-point EDSS decrease from baseline confirmed over 6 months (CDI is assessed only in patients with baseline EDSS score $\geqslant 2.0$ ). CDW is defined as $\geqslant 1$-point EDSS increase (or $\geqslant 1.5$ points if baseline EDSS $=0$ ) confirmed over 6 months.

(eTable 2 and Figure 4). A lower percentage of early relapsers was free of 6-month CDW and had achieved 6-month CDI compared with early non-relapsers over 6 years; early relapsers and early non-relapsers were free of MRI disease activity during Year 6 in comparable proportions, and the median BPF annual change at Year 6 was similar between the two groups (eTable 2 and Figure 4). The percentage of patients achieving annual NEDA during Year 6 was comparable between CARE-MS II early relapsers and early 


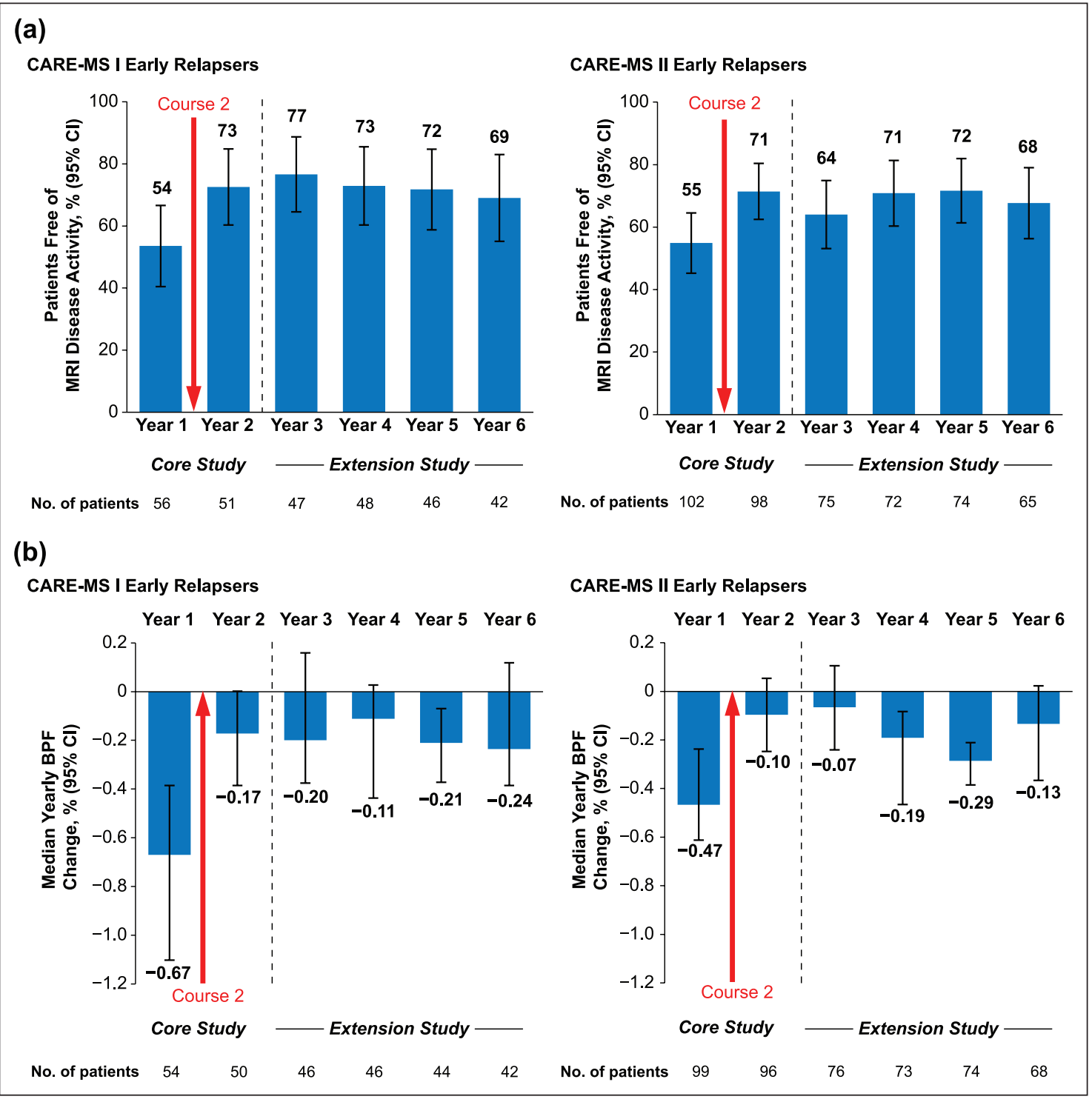

Figure 3. MRI outcomes in early relapsers through 6 years. (a) Proportions free of MRI disease activity and (b) median percentage yearly change in BPF over 6 years in patients with relapse between alemtuzumab Courses 1 and 2 in the core CARE-MS I and CARE-MS II studies. Freedom from MRI disease activity was defined as the absence of new gadolinium-enhancing T1 and new/enlarging T2 hyperintense lesions.

BPF: brain parenchymal fraction; CARE-MS: Comparison of Alemtuzumab and Rebif ${ }^{\circledR}$ Efficacy in Multiple Sclerosis; CIs: confidence intervals; MRI: magnetic resonance imaging.

Error bars denote $95 \%$ CIs.

non-relapsers but was slightly lower in the CARE-MS I early relapsing cohort (eTable 2).

\section{Discussion}

Limited practical guidance exists regarding management of patients with early on-treatment relapse following initiation of DMT. ${ }^{19}$ Relapse soon after treatment initiation may indicate suboptimal response, and a switch to an alternative DMT may be considered. ${ }^{19}$ The unique dosing regimen of alemtuzumab for treatment of relapsing forms of MS, consisting of two courses a year apart and additional courses as needed, includes a 12-month period in which patients have received a partial alemtuzumab dose, until they receive the second course. ${ }^{11,14}$ For some patients, the full immune modulatory effect may not be realized until after completing the two-course regimen. The results of this post hoc analysis indicate that patients who relapse within 12 months after the first alemtuzumab course may benefit from completing the treatment as approved, that is, by receiving the second course. 


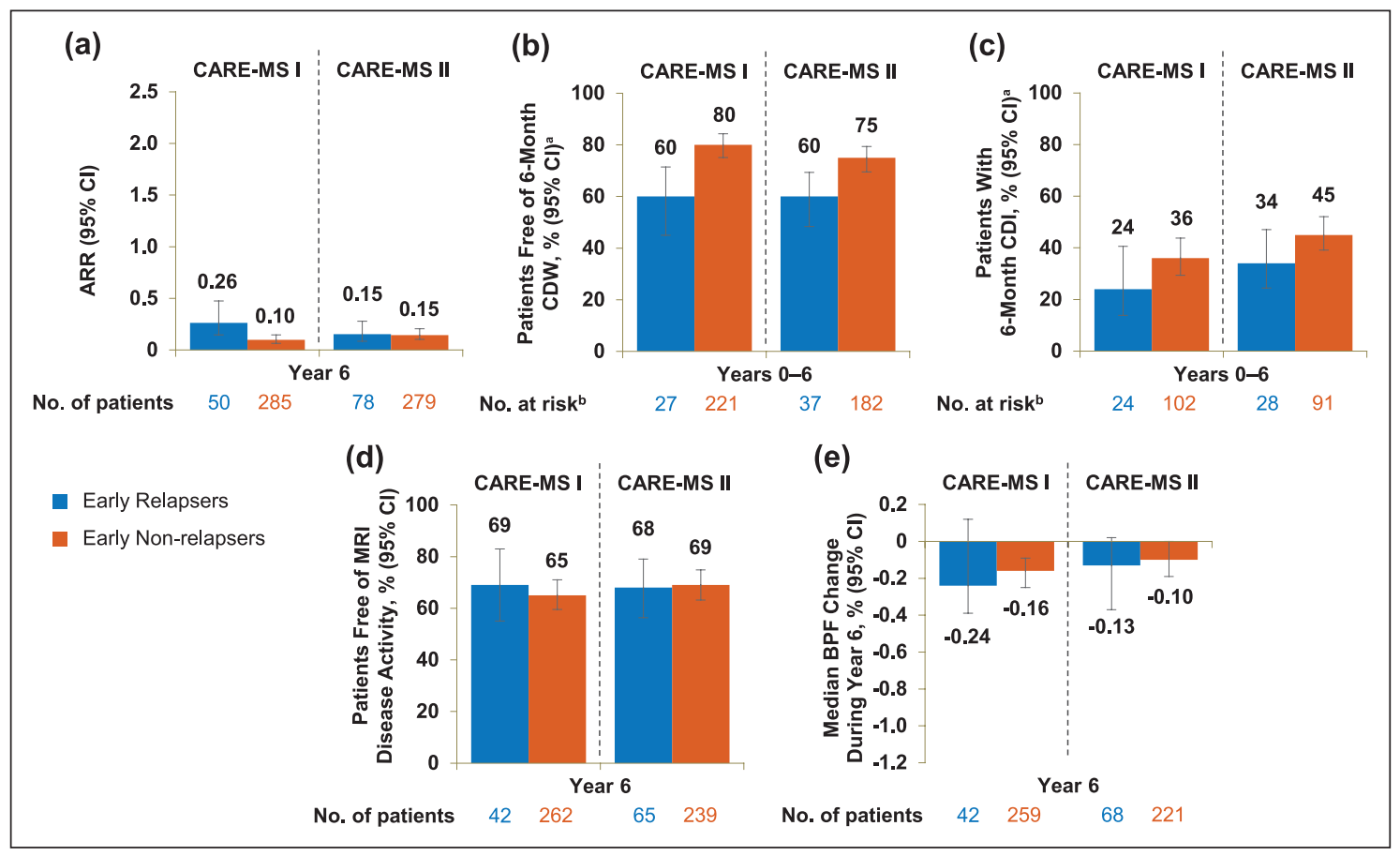

Figure 4. Efficacy outcomes of early relapsers and early non-relapsers through Year 6. (a) ARR during Year 6, (b) proportions free of 6-month CDW over 6 years, (c) proportions achieving 6-month CDI over 6 years, (d) proportions free of MRI disease activity during Year 6, and (e) median percentage change in BPF during Year 6 in patients with and without relapse between alemtuzumab Courses 1 and 2 in the core CARE-MS I and CARE-MS II studies. Freedom from MRI disease activity was defined as the absence of new gadolinium-enhancing T1 and new/enlarging T2 hyperintense lesions. ARR: annualized relapse rate; BPF: brain parenchymal fraction; CARE-MS: Comparison of Alemtuzumab and Rebif ${ }^{\mathbb{B}}$ Efficacy in Multiple Sclerosis; CDI: confirmed disability improvement; CDW: confirmed disability worsening; MRI: magnetic resonance imaging; CI: confidence interval; EDSS: Expanded Disability Status Scale.

Error bars denote $95 \%$ CIs.

aKaplan-Meier estimates.

${ }^{b}$ Number at risk is the number of patients who remained on study and had yet to experience 6-month CDW or 6-month CDI, respectively. $\mathrm{CDI}$ is defined as $\geqslant 1$-point EDSS decrease from baseline confirmed over 6 months (CDI is assessed only in patients with baseline EDSS score $\geqslant 2.0$ ). $\mathrm{CDW}$ is defined as $\geqslant 1$-point EDSS increase (or $\geqslant 1.5$ points if baseline EDSS $=0$ ) confirmed over 6 months.

After completion of the two-course regimen, clinical and MRI lesion outcomes were improved, despite clinical relapse after the first course. However, early relapsers responded to Course 1 of alemtuzumab, despite their relapse activity, as the percentage of patients free of Gd-enhancing lesions improved from baseline to Year 1 . The retention rates $(80 \%$ in CARE-MS I; $72 \%$ in CARE-MS II) among early relapsers lend strength to the dataset, although they were slightly lower than those of early non-relapsers at Year 6 ( $88 \%$ and $81 \%$, respectively).

In general, relapses are not uncommon in the first year of treatment with DMTs. For DMTs that are administered continuously, the percentages of patients relapsing within 12 months of treatment initiation were $32 \%-43 \%$ with SC IFNB-1a in the CARE-MS core studies, $17 \%$ with fingolimod in the TRANSFORMS study, ${ }^{16} 23 \%$ with natalizumab in the AFFIRM study, ${ }^{17}$ and $28 \%$ with intramuscular IFNB-1a and natalizumab combination therapy in the SENTINEL study. ${ }^{18}$ The proportions of patients with active RRMS relapsing within 12 months after the first alemtuzumab course in this study were $15 \%$ (CARE-MS I) and 24\% (CARE-MS II). Despite patient differences across studies of various DMTs, each therapy had a population with disease activity occurring shortly after initiation.

Disease activity soon after initiating a DMT does not necessarily represent treatment failure. ${ }^{20}$ Studies of other DMTs have shown that pretreatment relapse activity is predictive of on-treatment disease activity, including relapses, ${ }^{21-23}$ suggesting a higher underlying propensity for disease activity in some patients. This is supported in our study by the finding that, compared with patients without relapse between Courses 1 and 2, early relapsers had a higher mean number of relapses in the 1-2 years before study entry and a higher baseline mean EDSS score. 
Notably, a greater proportion of CARE-MS II patients relapsed within 12 months after the first alemtuzumab course compared with patients from CARE-MS I. Although pretreatment relapse activity was numerically similar among early relapsers in both studies, CARE-MS I enrollees had relapsed while treatment naive, whereas CARE-MS II patients had relapsed, despite prior therapy. The higher risk for early relapse in CARE-MS II patients is thus consistent with a greater baseline propensity for treatment-resistant MS disease activity in that study population. CARE-MS II enrollees also had a longer disease duration than CARE-MS I patients, but relapse rates tend to decrease over time,${ }^{24}$ suggesting that disease duration is unlikely to account for the observed difference in on-study relapses.

Early relapsers showed improvements in efficacy outcomes after Course 2 which were maintained through 6 years. These patients had similar MRI outcomes as early non-relapsers, but showed an increased risk for relapse and disability worsening, and decreased disability improvement. Moreover, fewer early relapsers achieved NEDA over 6 years compared with early non-relapsers. As early relapsers may have intrinsically more severe MS, they may need closer monitoring for clinical disease activity compared with patients who do not relapse between Course 1 and 2. However, reasonable disease control can often be achieved in these patients upon completion of the two-course regimen.

Alemtuzumab also slowed the annual rate of BVL in early relapsers over 6 years to rates almost as low as those of the overall CARE-MS population. ${ }^{4-6}$ Given the link between brain atrophy and long-term disability, ${ }^{25}$ the slowing effect of alemtuzumab on BVL should be expected to minimize future disability worsening even in patients who relapsed after the first course.

During the 4-year extension study, early relapsers were approximately 1.5 times more likely to receive a third alemtuzumab course and, on average, received it 5 months sooner, compared with early non-relapsers. Higher retreatment rates in this subgroup reflect the protocol requirement for disease activity as a condition of eligibility for receiving additional alemtuzumab courses in the extension. Nevertheless, 54\% (CARE-MS I) and 40\% (CARE-MS II) of early relapsers did not receive additional alemtuzumab after Course 2, and $90 \%$ and $86 \%$ did not receive another DMT, suggesting that disease activity was sufficiently controlled in many of these patients after completion of the two-course regimen.
Treatment sequencing has potential implications for disease activity after the first alemtuzumab course. A retrospective analysis of 50 patients transitioning from fingolimod to alemtuzumab showed a strong reduction in disease activity within 12 months after the switch. ${ }^{26}$ However, in another series of patients treated sequentially with fingolimod and then alemtuzumab, nine were identified as experiencing disease activity within 12 months after alemtuzumab Course $1 .{ }^{27}$ Even though animal studies showed some degree of lymphocyte depletion in lymph nodes after alemtuzumab administration, ${ }^{8}$ the authors speculated that residual sequestration in lymph nodes after cessation of fingolimod treatment could protect lymphocytes from alemtuzumab-mediated depletion and contribute to persistence of disease activity. ${ }^{27}$ Importantly, eight of those nine patients were relapse free after completing the two-course regimen. In the CARE-MS studies, no patients had a treatment history of fingolimod before enrollment. Nonetheless, an appropriate washout period may be advisable when transitioning to alemtuzumab from other agents with lymphopenic effects. ${ }^{28}$

A limitation of this post hoc study is that the early relapsing and early non-relapsing subgroups were retrospectively defined and differed on multiple measures of MS disease severity at baseline, including relapse frequency before the first alemtuzumab course. The substantial difference in the size of the two subgroups (early relapsers, $n=161$; early non-relapsers, $n=650$ ) may further confound statistical comparisons. Thus, comparisons between these groups must be interpreted with caution. A second limitation is that this study was not designed to evaluate the effectiveness of switching to another therapy in comparison with receiving the second alemtuzumab course.

\section{Conclusion}

Alemtuzumab, administered in two annual courses, constitutes a unique treatment approach that does not require regular dosing to achieve and maintain efficacy. ${ }^{4-6,28}$ Some patients may relapse between the two initial alemtuzumab courses, but improved outcomes following Course 2 suggest that the two-course regimen, per the approved labeling, could maximize clinical and MRI benefits.

\section{Acknowledgements}

The authors and Sanofi would like to thank the patients for their participation in the trials, as well as the CARE-MS I and CARE-MS II Steering Committees and CAMMS03409 Investigators. This manuscript was reviewed for scientific accuracy by Darren P Baker, $\mathrm{PhD}$, Ericka M Bueno, PhD, Jordan Messer, PharmD, 
and Colin Mitchell, PhD, of Sanofi. Statistical support was provided by Nadia Daizadeh, PhD, of Sanofi. Editorial support was provided by Panos Xenopoulos, $\mathrm{PhD}$, and Valerie P Zediak, PhD, of Eloquent Scientific Solutions, and was funded by Sanofi.

\section{Declaration of Conflicting Interests}

The author(s) declared the following potential conflicts of interest with respect to the research, authorship, and/or publication of this article: B.V.W. received research and travel grants; honoraria for MS-expert advice; and speakers' fees from Actelion, Bayer Schering, Biogen, Merck Serono, Novartis, Roche, Sanofi, and Teva. B.A.S. received fees for speaking and/or consulting from AbbVie, Acorda, Biogen, EMD Serono, Genentech, Novartis, Pfizer, Roche, Sanofi, and Teva and research support from AbbVie, Acorda, Alkermes, Biogen, MedImmune, Novartis, Roche, and Sanofi. A.B. received consulting fees and/or fees for non-CME services from Biogen, Mallinckrodt, Medtronic, Novartis, Sanofi, and Teva. S.B. received speaking and/or consulting fees from Bayer, Biogen, Merck Serono, Novartis, Roche, and Sanofi. O.F. received speaking and/or consulting fees from Allergan, Almirall, Bayer Schering, Biogen, Merck Serono, Novartis, Sanofi, and Teva; compensation for serving as a journal editor, associate editor, or member of an editorial advisory board for Revista Española de Esclerosis Múltiple; and research support from the Hospital Foundatión FIMABIS. M.S.F. received honoraria/consulting fees from Actelion, Bayer, Biogen, Canada Innovation, Chugai, EMD Canada, Merck Serono, Novartis, Roche, Sanofi, and Teva; support as a member of advisory board, board of directors, or other similar group from Actelion, Bayer, Biogen, Clene, Merck Serono, Novartis, Roche, and Sanofi; and fees for participation in speaker's bureau from Sanofi. G.I. received speaking and advisory fees from Almirall, Bayer, Biogen, Merck Serono, Novartis, Roche, Sanofi, and Teva. J.L. received travel support and/or lecture honoraria from Biogen, Novartis, Sanofi, and Teva; support from scientific advisory boards from Almirall, Biogen, Novartis, Sanofi, and Teva; compensation as part of the editorial board for Acta Neurologica Scandinavica; and unconditional research grants from Biogen, Novartis, and Teva. C.P. received consulting and/or speaking fees, research, and travel grants from Actelion, Biogen, Merck, Novartis, Sanofi, and Teva. B. Sharrack received research and travel grants; honoraria for expert advice on MS; and speaker's fees from Biogen, Merck, Novartis, Roche, Sanofi, and Teva. B. Steingo received consulting; speaking fees; and/or grant/research support from Acorda, Biogen, EMD Serono, Mallinckrodt, Novartis, Sanofi, and Teva. H.W. received consulting and/or speaking fees and grant/research support from Bayer, Bayer Schering Pharma, Biogen, Elan Corporation, Lilly, Lundbeck, Merck Serono, Novartis, Novo Nordisk, Sanofi, and Teva Neuroscience. S.W. received fees as consultant; principal investigator; and/or speaker from Alkermes, Asclepios, Biogen, Celgene, EMD Serono, Novartis, Roche, Sanofi, and TG Therapeutics. T.Z. received consulting and/or speaking fees from Almirall, Bayer, Biogen, Merck, Novartis, Roche, Sanofi, and Teva and grant/research support from Biogen, Novartis, Sanofi, and Teva. L.C. and K.T. receive compensation as employees of Sanofi. D.H.M. received compensation as an employee of Sanofi at the time this study was conducted and is currently an employee of Cerevance, Inc. P.V. received consulting and/or speaking fees and research support from Almirall, Bayer, Biogen, Celgene, Merck Serono, Novartis, Sanofi, Servier, and Teva.

\section{Funding}

The author(s) disclosed receipt of the following financial support for the research, authorship, and/or publication of this article: The CARE-MS I, CARE-MS II, and CAMMS03409 studies were funded by Sanofi and Bayer HealthCare Pharmaceuticals. Only Sanofi contributed to the conduct of the studies; collection, management, analysis, and interpretation of the data; review of the manuscript. Apart from the Sanofi employees listed as authors, the funders had no role in preparation, approval, and decision to submit the manuscript for publication.

\section{ORCID iDs}

Simon Broadley (iD https://orcid.org/0000-0002 $-9429-4307$

Tjalf Ziemssen (iD https://orcid.org/0000-0001-8799 $-8202$

\section{Supplemental material}

Supplemental material for this article is available online.

\section{References}

1. Cohen JA, Coles AJ, Arnold DL, et al. Alemtuzumab versus interferon beta 1a as first-line treatment for patients with relapsing-remitting multiple sclerosis: A randomised controlled phase 3 trial. Lancet 2012; 380(9856): 1819-1828.

2. Coles AJ, Twyman CL, Arnold DL, et al. Alemtuzumab for patients with relapsing multiple sclerosis after disease-modifying therapy: A randomised controlled phase 3 trial. Lancet 2012; 380(9856): 1829-1839. 
3. Giovannoni G, Cohen JA, Coles AJ, et al. Alemtuzumab improves preexisting disability in active relapsing-remitting MS patients. Neurology 2016; 87(19): 1985-1992.

4. Coles AJ, Cohen JA, Fox EJ, et al. Alemtuzumab CARE-MS II 5-year follow-up: Efficacy and safety findings. Neurology 2017; 89: 1117-1126.

5. Havrdova E, Arnold DL, Cohen JA, et al. Alemtuzumab CARE-MS I 5-year follow-up: Durable efficacy in the absence of continuous MS therapy. Neurology 2017; 89: 1107-1116.

6. Ziemssen $\mathrm{T}$ and Thomas K. Alemtuzumab in the long-term treatment of relapsing-remitting multiple sclerosis: An update on the clinical trial evidence and data from the real world. Ther Adv Neurol Disord 2017; 10(10): 343-359.

7. Cox AL, Thompson SA, Jones JL, et al. Lymphocyte homeostasis following therapeutic lymphocyte depletion in multiple sclerosis. Eur J Immunol 2005; 35(11): 3332-3342.

8. $\mathrm{Hu} \mathrm{Y}$, Turner MJ, Shields J, et al. Investigation of the mechanism of action of alemtuzumab in a human CD52 transgenic mouse model. Immunology 2009; 128(2): 260-270.

9. Wiendl H, Bourdette D and Ciccarelli O. Can immune reprogramming with alemtuzumab induce permanent remission in multiple sclerosis. Neurology 2017; 89(11): 1098-1100.

10. Wray S, Havrdova E, Snydman DR, et al. Infection risk with alemtuzumab decreases over time: Pooled analysis of 6-year data from the CAMMS223, CARE-MS I, and CARE-MS II studies and the CAMMS03409 extension study. Mult Scler 2019; 25(12): 1605-1617.

11. LEMTRADA [Summary of Product Characteristics], 2019. Diegem; Belgium: Sanofi Belgium.

12. Phelps R, Winston JA, Wynn D, et al. Incidence, management, and outcomes of autoimmune nephropathies following alemtuzumab treatment in patients with multiple sclerosis. Mult Scler 2019; 25(9): 1273-1288.

13. Cuker A, Bass AD, Nadj C, et al. Immune thrombocytopenia in alemtuzumab-treated MS patients: Incidence, detection, and management. Mult Scler. Epub ahead of print 20 February 2019. DOI: $10.1177 / 1352458518816612$.

14. LEMTRADA® (alemtuzumab) injection for intravenous use [prescribing information], 2019. Cambridge, MA: Genzyme Corporation.

Visit SAGE journals online journals.sagepub.com/ home/msj

(SAGE journals
$1 \& \mathrm{~d}=2018051116114622483$ (accessed 17 December 2018).

16. Cohen JA, Barkhof F, Comi G, et al. Oral fingolimod or intramuscular interferon for relapsing multiple sclerosis. N Engl J Med 2010; 362: 402-415.

17. Polman CH, O'Connor PW, Havrdova E, et al. A randomized, placebo-controlled trial of natalizumab for relapsing multiple sclerosis. N Engl J Med 2006; 354(9): 899-910.

18. Rudick RA, Stuart WH, Calabresi PA, et al. Natalizumab plus interferon beta-1a for relapsing multiple sclerosis. N Engl J Med 2006; 354: 911-923.

19. Gajofatto A and Benedetti MD. Treatment strategies for multiple sclerosis: When to start, when to change, when to stop. World J Clin Cases 2015; 3(7): $545-555$.

20. Hesse D, Krakauer M, Lund H, et al. Breakthrough disease during interferon-[beta] therapy in MS: No signs of impaired biologic response. Neurology 2010; 74(18): 1455-1462.

21. Kalincik T, Manouchehrinia A, Sobisek L, et al. Towards personalized therapy for multiple sclerosis: Prediction of individual treatment response. Brain 2017; 140(9): 2426-2443.

22. Laroni A, Gandoglia I, Solaro C, et al. Clinical baseline factors predict response to natalizumab: Their usefulness in patient selection. BMC Neurol 2014; 14: 103.

23. Giuliani M, Logoteta A, Prosperini A, et al. Baseline characteristics associated with NEDA-3 status in fingolimod-treated patients with relapsing-remitting multiple sclerosis. Mult Scler Demyelinating Disord 2017; 2: 10.

24. Tremlett H, Zhao Y, Joseph J, et al. Relapses in multiple sclerosis are age- and time-dependent. J Neurol Neurosurg Psychiatry 2008; 79(12): 1368-1374.

25. Popescu V, Agosta F, Hulst HE, et al. Brain atrophy and lesion load predict long term disability in multiple sclerosis. J Neurol Neurosurg Psychiatry 2013; 84(10): 1082-1091.

26. Huhn K, Bayas A, Doerck S, et al. Alemtuzumab as rescue therapy in a cohort of 50 relapsing-remitting MS patients with breakthrough disease on fingolimod: A multi-center observational study. J Neurol 2018; 265(7): 1521-1527.

27. Willis M, Pearson O, Illes Z, et al. An observational study of alemtuzumab following fingolimod for multiple sclerosis. Neurol Neuroimmunol Neuroinflamm 2017; 4(2): e320.

28. Berger T, Elovaara I, Fredrikson S, et al. Alemtuzumab use in clinical practice: Recommendations from European multiple sclerosis experts. CNS Drugs 2017; 31(1): 33-50. 